\title{
BLOCKCHAIN-BASED NETWORK FUNCTIONS VIRTUALIZATION FOR 5G NETWORK SLICING
}

\author{
Taras MAKSYMYUK*, Volodymyr ANDRUSHCHAK*, Stepan DUMYCH*, Bohdan SHUBYN*, \\ Gabriel BUGÁR**, Juraj GAZDA** \\ *Department of Telecommunications, Institute of Telecommunications, Radio Electronics and Electronic Engineering, Lviv \\ Polytechnic National University, Bandery 12, 79013 Lviv, Ukraine, tel. +380633299117, \\ E-mail:taras.maksymyuk@gmail.com, volodya1andrushchak@gmail.com, stiv.dumych@ gmail.com, boshubin@gmail.com, \\ ${ }^{* *}$ Department of Computers and Informatics, Technical University of Košice, Letná 9, 04200 Košice, Slovakia, \\ E-mail: gabriel.bugar@tuke.sk, juraj.gazda@tuke.sk
}

\begin{abstract}
The paper proposes a new blockchain-based network architecture for $5 G$ network functions virtualization. By using a combination of AI and blockchain technologies, proposed system provides flexible network deployment, interoperability between different mobile network operators and effective management of radio resources. Experimental testbed of the proposed system has been implemented by using cloud and edge computing infrastructure and software defined radio peripheral NI USRP 2900. Simulation results of the network slicing and radio resource management shows that proposed system is able to double the capacity of the physical network infrastructure, while ensuring the target quality of service for all users.
\end{abstract}

Keywords: 5G, blockchain, AI, SDN, virtualization

\section{INTRODUCTION}

With the advent of $5 \mathrm{G}$ trend, mobile networks became even more heterogeneous in terms of transmission range, data rates, capacity and energy efficiency. 5G essentially became a buzzword for any radio access technology, which has emerged after 3GPP Release 10, such as small cells, device-to-device communications, massive MIMO (multiple input multiple output) antenna arrays, new modulation waveforms, LTE-unlicensed, narrowband-IoT, mmWaves and many others [1]. This essentially introduced a large number of additional degrees of freedom for the end users in terms of connectivity options. These additional degrees of freedom substantially increase the complexity of network optimization, management and maintenance for the operators. To tackle with this problem, $5 \mathrm{G}$ has adopted the concept of software defined networking, network functions virtualization and intelligent network management to provide more agile and flexible infrastructure [2].

Further evolution beyond $5 \mathrm{G}$ is definitely not going to simplify network deployment, so it is wise enough to expect new technologies, which are not yet feasible for $5 \mathrm{G}$, to be included in one of future $6 \mathrm{G}$ marketed 3GPP releases [3]. The preliminary requirements for $6 \mathrm{G}$ are targeting terabit data rates per user, less than one millisecond latency, several orders of magnitude higher overall capacity, improved energy efficiency and strong security [4]. 6G will likely support applications far beyond anything seen so far, ranging from ultra-low energy IoT to immersive user experience with mixed reality and tactile internet [5]. The density of the connected devices will exponentially grow following by even higher densification of the network infrastructure. Whereas $5 \mathrm{G}$ is mainly the system of thousands femtocells, 6G will probably be the system of millions attocells, with range up to ten meters and capacity of several terabits per second. Thus, the role of artificial intelligence (AI) in 6G will be even more crucial in order to provide efficient network operation in complex hyper-dense deployment scenarios [6].
This article introduces a new vision on the future mobile network ecosystem beyond 5G. We propose a novel blockchain-based architecture for decentralized deployment and intelligent management of the virtualized network functions. Blockchain is the distributed ledger technology that provides a scalable decentralized platform to automate interaction between different entities in trustful and secure manner [7]. The features of blockchain make it useful for mobile network operation to simplify user authentication, handover, network slicing, end-to-end quality control and many other network functions.

This paper is organized as follows. Section 2 describes the proposed design for converged network infrastructure. Section 3 provides simulation results of the network performance. Section 4 concludes the paper.

\section{INTELLIGENT BLOCKCHAIN-BASED VIRTU- ALIZED 5G NETWORK ARCHITECTURE}

\subsection{Key Features of the Blockchain technology}

Blockchain is the distributed ledger that can permanently record and verify transactions between multiple parties [8]. The simple example of the transaction is the event when end user sends the payment to the MNO for the service with specified SLA (Service Level Agreement). All transactions are stored in the blocks, which are linked together as a chain. The blockchain is decentralized, so that all nodes keep the copy of the whole chain or at least several latest blocks. Each block contains the data about multiple transactions, and additional header which contains a hash of the previous block, so that any block is a dependent on the all previous blocks in the chain. A hash is a unique cryptographic identity that ensures integrity and correctness of the information in the block. New blocks are added to blockchain by the computationally complex procedure of hash calculation called mining. When new block is added to the blockchain, all other nodes must verify it by reaching the consensus. Therefore, any attempt to modify 
past transactions (e.g. SLA) will change the hash of the all subsequent blocks, so that other nodes will detect and decline the wrong blocks. Thus, to modify information in the blockchain, malicious node would need to calculate hashes of all subsequent blocks, which is not possible because new blocks with correct hashes will be continually added and malicious node will never catch the main chain. This features allows to use blockchain a core technology for decentralized mobile networks to orchestrate virtual network functions among multiple operators and service slices with trustfully and secure data management.

\subsection{Blockchain and AI-Empowered Virtualized 5G Network Architecture}

Unlike previous generations of the 3GPP standards family, an architecture of 5G networks is mostly service based. This means that most of architecture elements are defined as separate network functions, which are available via common framework to any entity with corresponding access rights. By using a shared network repository functions (NRF), any network function can use the services offered by other network functions. Such architecture model supports modularity, reusability and self-containment of network functions that allows to take advantage of the latest virtualization and software technologies. To further extend the functionality of the architecture, defined in 3GPP TS 23.501 [9], in this paper we propose an new multilayer network architecture, which is aimed to facilitate intelligent automated network management by introducing a blockchain middleware (Fig.1). The main reason of blockchain in the current context is in the utilization of the distributed ledger technology as a decentralized database to support data exchange between virtualized network functions [10].

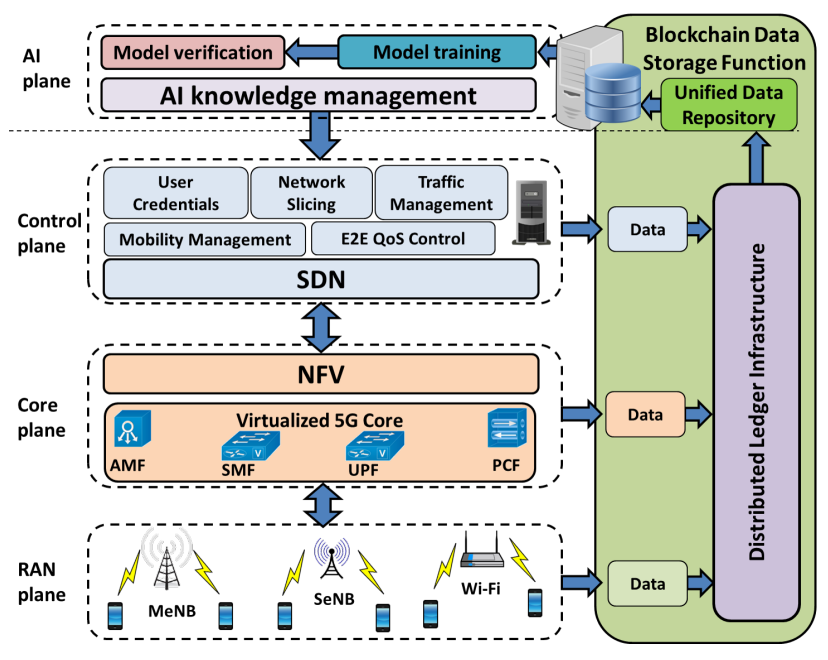

Fig. 1 Blockchain and AI-empowered virtualized 5G network arhitecture

In the proposed architecture, all planes are logically separated according to their functional responsibilities but interact with each other over the blockchain middleware. More detailed description of each plane is provided below.
The RAN (Radio Access Network) plane consists of macro and small cells, Wi-Fi access points, and other connectivity infrastructure. Although, this plane, encompasses all the functionality of signal processing, users scheduling and wireless transmission, the high level configurations of the transceivers are determined by higher planes.

Considering the major changes, which $5 \mathrm{G}$ brings comparing to the LTE the functionality of core network should revisited. Since all end-to-end services in $5 \mathrm{G}$ are created from virtualized service functions, there are key differences in functionality that results in the new paradigm Network Slicing as a Service according to the following rules:

1. Service orchestration and slice management need to be application aware.

2. Service orchestration and slice management need to be quality aware

3. Control plane is aware of the slices but not of their specific services.

Furthermore, network slicing enables new market opportunities for separate special requirements of different verticals. In addition, service functions and network components can be created by different vendors and operators among multiple network domains and locations. Functionality of blockchain in the proposed 5G architecture allows network functions to securely store their contexts by using Data Storage Functions (DSF).

Unlike previous system architectures, which had more persistent UE associations in the home PLMN (Public Land Mobile Network), 5G enables more convenient change of the UEs serving by using new AMF (Access and Mobility Management Functions) functionality. In difference to LTE MME (Mobility Management Entity), AMF instances are fully virtualized that allows UE to switch between different AMFs of any operator in a matter of seconds. To support such dynamic migration, the important contextual information of the UE is stored in a common data repository and managed by distributed ledgers in a blockchain to provide security and verification in a decentralized manner. Each $\mathrm{UE}$ in this case used its unique identifier in a blockchain, which is similar to the unique bitcoin wallet address and can be accessed by all nodes in the network. Such architecture allows to improve service resilience and load balancing by allowing any AMF to serve any UE.

Control plane is mainly responsible for softwaredefined network (SDN) management by using advanced algorithms of load balancing, spectrum sharing, network slicing, end-to-end (E2E) QoS (Quality of Service) control, etc. To enable effective network management SDN controller leverages the massive amount of acquired network information by monitoring all network nodes simultaneously, including both network side equipment and user side equipment. To solve this important problem we incorporate blockchain as an immutable decentralized database that can track all rapidly variable changes of network conditions. Control plane acts as an intermediary between AI plane and core plane by applying the inferences and decisions made by machine learning models to the real network configuration such as load balancing, RAN reconfiguration and mobility management in order to satisfy E2E QoS requirements of all network slices. 
Finally, AI plane consists of high-performance servers and databases, which are used to collect and process the data. The important function of knowledge plane to derive the intelligence from acquired data according to the pretrained AI algorithms. For example, AI plane is able to detect when network performance is becoming worse when something changes in the channels allocation, severe interference appears or some cells are facing traffic overloads.

\section{SIMULATIONS AND PERFORMANCE ANALY- SIS}

\subsection{Implementation of the software defined mobile network testbed based on the virtualized network functions}

Based on the virtualized network architecture we implement the logical network split into several slices with different E2E QoS requirements. All logical slices are isolated from each other in terms of access to the physical resources of the $5 \mathrm{G}$ infrastructure [11]. In order to perform network slicing, we implement the real testbed of mobile communication system based on the 5G-EmPOWER operating system, which allows to separate the RAN and control planes [12]. The interaction between RAN and control plane is conducted based on the OpenEmpower protocol. Control plane and core planes are completely virtualized based on the edge computing infrastructure and connected to a powerful cloud-based AI plane. Physical gNB is implemented by using a software-defined radio NI USRP 2900, which is connected to the $5 \mathrm{G}$ core. Thus, the deployed virtualized network infrastructure includes the powerful AI infrastructure, the 5G-EmPOWER SDN controller, the 5G virtualized core, and the virtual gNB agent for the base station (Fig. 2). The developed testbed is flexible and supports multiple services, including network slicing with isolation of network functions. 5G-Empower agents interact with the controller to ensure load balancing and physical resource blocks allocation between network slices.

Existing 5G-EmPOWER interface does not allow to change the RAN parameters remotely, so that each gNB configuration should be stored as a corresponding file in the edge server. Therefore, in this paper we implement the principle of digital twins by creating the corresponding configuration files for each gNB in the cloud. The configuration files then synchronized between cloud and core edge server in the same way how traditional cloud storage works. Such solution allows to simplify configuration of the radio access network based on data from the AI plane. Note that all virtual network functions are deployed in isolated Docker containers, which contains the following network functions:

- AMF (Access and Mobility Management Function) to control user admission and mobility.

- SMF (Session Management Function) to control user sessions.

- UPF (User Plane Function) to manage data transmission in uplink and downlink direction for end users.

- PCF (Policy Control Function) to manage the higher level QoS policies.

\subsection{Practical implementation of 5G network slicing}

By using the 5G-EmPOWER operating system we deploy multiple virtualized slices on top of a single physical infrastructure. Such an approach ensures efficient use of network resources while maintaining the target E2E QoS requirements. Network slices are created based on descriptors that the virtual mobile network operator passes to the physical mobile infrastructure operator according to the corresponding SLA. All SLAs between operators and users are validated in blockchain and verified by all operators by using delegated proof-of-stake consensus protocol. Thus, each UE is assigned to one or more network slices with the corresponding SLA(s). The 5G-EmPOWER operating system supports a flexible radio resource management mechanism between individual network slices by using SRM (Slice Resource Manager) function, which manages the life cycle of network slices for each eNB. After receiving a new request for slice creation, SRM checks the resource availability within the current infrastructure. If the number of PRB (Physical Resource Block) is sufficient for new slice, it will be created and the corresponding access rights will be granted to UEs. Otherwise, SRM will either decline the creation of the slice or create it with reduced radio resources, depending on the initial conditions of the request.

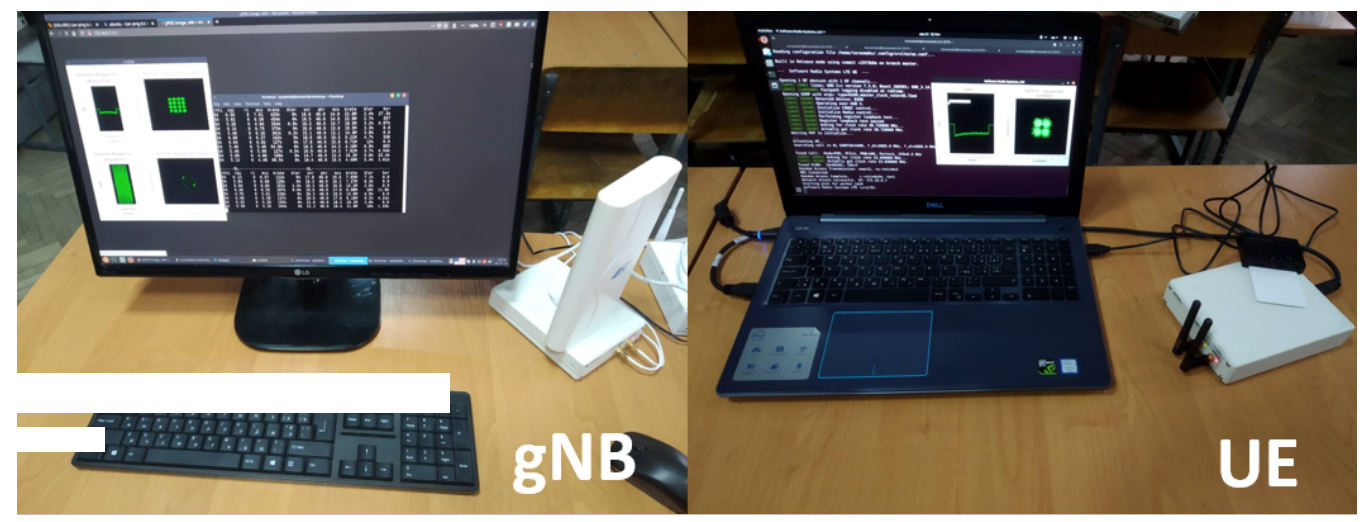

Fig. 2 Experimental testbed of the virtualized 5G network based on software defined radio 


\subsection{Simulation results and discussion}

Technically, blockchain keeps all records about spectrum allocation and overall network performance, which can be used to train AI-engine. First, the history on traffic demand variation is acquired from the blockchain for each target $\mathrm{gNB}$. These data are represented in the timeseries and used to train the AI prediction model, which is based on the LSTM (Long Short-Term Memory) recurrent neural network. When reaching loss criteria within LSTM optimization framework, operator is able to predict traffic demand for a target timeframe. The detailed analysis of the LSTM performance is provided in our preliminary research, where we have used traffic prediction for spectrum allocation in HetNet and achieved $90 \%$ accuracy on the traffic prediction (Fig.3) [13].

In order to simulate the adaptive resource management among network slices, we develop the framework, where dataset on the multiple traffic loads is feeded to the deep learning model [14].
Simulation results show that when traffic load for one slice is lower, the capacity is redistributed among other network slices, so that the physical resources of the mobile network are efficiently distributed among the users. In Fig.4, the red line resembles static resource allocation equally between all network slices. In this case, when traffic demand increases for the network slice 1, part of the users cannot be served by this slice that decreases overall network performance. In difference to the static resource allocation, proposed deep learning approach based on the LSTM recurrent neural networks can predict the traffic demand for each slice with high accuracy and send the corresponding request to the SRM through blockchain infrastructure to change the bandwidth of each slice proportionally to the predicted demand. The simulation result for the AI-based network slicing is reflected by blue line in the Fig.4. As observed, during the peak network load, proposed AI-based network slicing allows to increase total network capacity almost twice, comparing to the static solution.

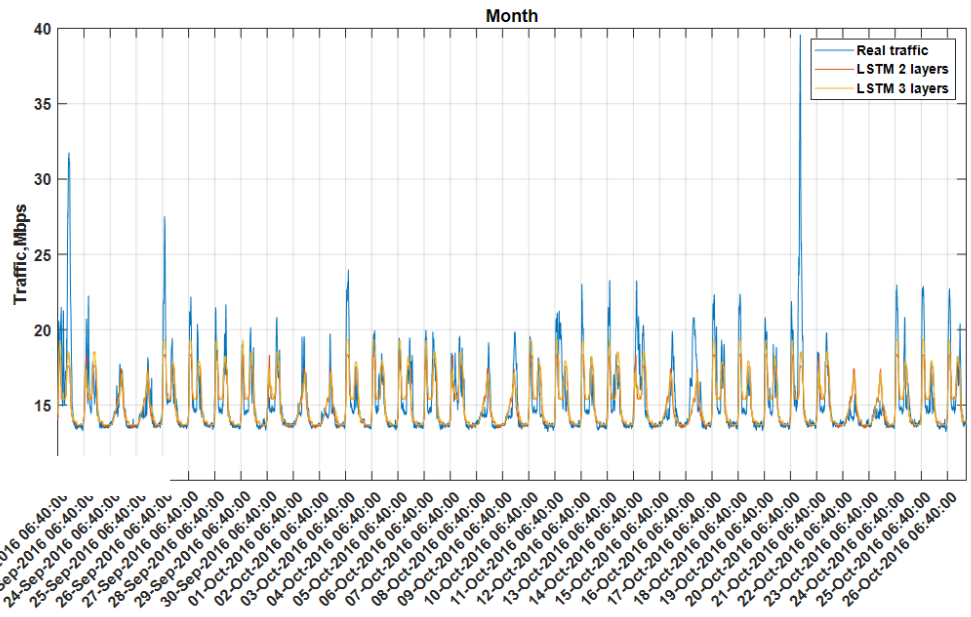

Fig. 3 Simulation results of the LSTM-based traffic prediction

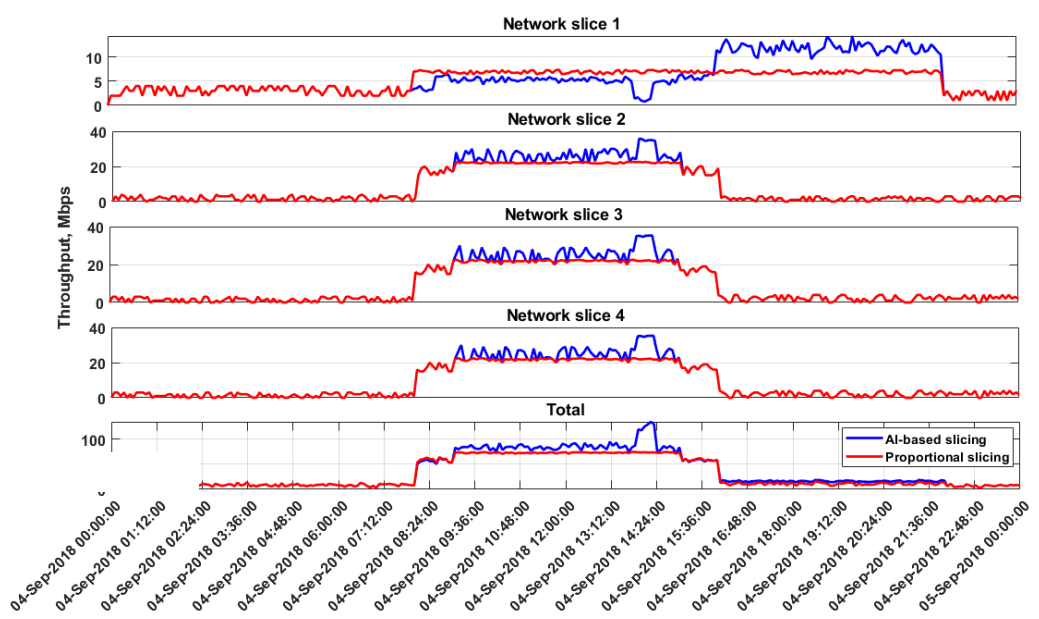

Fig. 4 Simulation results of the AI-based 5G network slicing based on the virtualized network architecture 


\section{CONCLUSIONS}

In this paper, we have proposed a novel blockchainbased architecture for $5 \mathrm{G}$ network function virtualization. Proposed architecture allows to improve the flexibility of network deployment by using direct interaction between any virtualized network instances of any mobile network operator with any end user in a secure manner by using distributed ledger infrastructure in a blockchain. Based on the proposed architecture, a practical testbed is developed based on the software-defined radio and 5G-Empower network operating system. Simulation results have been conducted to show how the proposed $5 \mathrm{G}$ architecture can leverage the potential of blockchain and AI interaction to improve the performance of network slicing by predicting the traffic demand of each network slice. According to the simulation results, proposed solution provides almost 2 times higher overall throughput of the physical network infrastructure during peak traffic load.

\section{ACKNOWLEDGEMENT}

This research was supported by the Ukrainian government project 0120U100674, Designing the novel decentralized mobile network based on blockchain architecture and artificial intelligence for $5 \mathrm{G} / 6 \mathrm{G}$ development in Ukraine, by the Slovak Research and Development Agency, project numbers APVV-18-0214 and APVV-18-0368, and by the Scientific Grant Agency of the Ministry of Education, Science, Research and Sport of Slovakia under the contract $1 / 0268 / 19$

\section{REFERENCES}

[1] A. OSSEIRAN, J.F. MONSERRAT, P. MARSCH, M. DOHLER, and T. NAKAMURA. $5 G$ Mobile and Wireless Communications Technology. Cambridge University Press, 2016.

[2] N. ZHANG, P. YANG, S. ZHANG, D. CHEN, W. ZHUANG, B. LIANG, and X. S. SHEN. Software defined networking enabled wireless network virtualization: Challenges and solutions. IEEE Network, 31(5):42-49, 2017.

[3] P. YANG, Y. XIAO, M. XIAO, and S. LI. 6g wireless communications: Vision and potential techniques. IEEE Network, 33(4):70-75, 2019.

[4] Z. ZHANG, Y. XIAO, Z. MA, M. XIAO, Z. DING, X. LEI, G. K. KARAGIANNIDIS, and P. FAN. $6 \mathrm{~g}$ wireless networks: Vision, requirements, architecture, and key technologies. IEEE Vehicular Technology Magazine, 14(3):28-41, 2019.

[5] L. ZHANG, Y. LIANG, and D. NIYATO. $6 \mathrm{~g}$ visions: Mobile ultra-broadband, super internet-ofthings, and artificial intelligence. China Communications, 16(8):1-14, 2019.

[6] K. B. LETAIEF, W. CHEN, Y. SHI, J. ZHANG, and Y. A. ZHANG. The roadmap to 6g: Ai empowered wireless networks. IEEE Communications Magazine, 57(8):84-90, 2019.

[7] B. MAFAKHERI, T. SUBRAMANYA, L. GORATTI, and R. RIGGIO. Blockchain-based infrastructure sharing in $5 \mathrm{~g}$ small cell networks. In 2018 14th International Conference on Network and Service Management (CNSM), pages 313-317, 2018.

[8] T. T. A. DINH, R. LIU, M. ZHANG, G. CHEN, B. C. OOI, and J. WANG. Untangling blockchain: A data processing view of blockchain systems. IEEE Transactions on Knowledge and Data Engineering, 30(7):1366-1385, 2018.

[9] M. SHIN, S. LEE, S. LEE, and D. KIM. A way forward for accommodating $\mathrm{nfv}$ in $3 \mathrm{gpp} 5 \mathrm{~g}$ systems. In 2017 International Conference on Information and Communication Technology Convergence (ICTC), pages 114-116, 2017.

[10] T. MAKSYMYUK, J. GAZDA, M. VOLOSIN, G. BUGAR, D. HORVATH, M. KLYMASH, and M. DOHLER. Blockchain-empowered framework for decentralized network management in 6g. IEEE Communications Magazine, 58(9):86-92, 2020.

[11] W. LEE, T. NA, and J. KIM. How to create a network slice? - a 5g core network perspective. In 2019 21st International Conference on Advanced Communication Technology (ICACT), pages 616-619, 2019.

[12] E. CORONADO, S. N. KHAN, and R. RIGGIO. 5gempower: A software-defined networking platform for $5 \mathrm{~g}$ radio access networks. IEEE Transactions on Network and Service Management, 16(2):715-728, 2019.

[13] T. MAKSYMYUK, L. HAN, S. LARIONOV, B. SHUBYN, A. LUNTOVSKY, and M. KLYMASH. Intelligent spectrum management in $5 \mathrm{~g}$ mobile networks based on recurrent neural networks. In 2019 IEEE 15th International Conference on the Experience of Designing and Application of CAD Systems (CADSM), pages 1-4, 2019.

[14] T. MAKSYMYUK, J. GAZDA, M. RUZICKA, E. SLAPAK, G. BUGAR, and L. HAN. Deep learning based mobile network management for $5 \mathrm{~g}$ and beyond. In 2020 IEEE 15th International Conference on Advanced Trends in Radioelectronics, Telecommunications and Computer Engineering (TCSET), pages 890-893, 2020.

Received December 12, 2020, accepted January 13, 2021

\section{BIOGRAPHIES}

Taras Maksymyuk received the B.A. degree in telecommunications in 2010, the M.S. degree in information communication networks in 2011, and the Ph.D. degree in telecommunication systems and networks in 2015 , all from 
the Lviv Polytechnic National University. He is currently a Senior Lecturer with the Telecommunications Department, Lviv Polytechnic National University, Lviv, Ukraine. He did his post-doc fellowship in Internet of Things and Artificial Intelligence Lab, Korea University under supervision of Prof. Minho Jo. He was recognized as the Best Young Scientist of Lviv Polytechnic National University in 2015, received the Lviv State Administration prize for outstanding scientific achievements and contribution in 2016, and Lviv Metropolitan Prize for best scientists in 2017. He is currently an Associate Editor of IEEE Comm. Magazine, and an Editor of the International Journal of Internet of Things and Big Data. His research interests include cognitive radio networks, LTE in unlicensed spectrum, mobile cloud computing, blockchain, 5G heterogeneous networks, software-defined radio access networks, Internet of Things, Big Data and artificial intelligence.

Volodymyr Andrushchak received the B.A. degree in telecommunications in 2015 and the M.S. degree in information communication networks in 2016, all from the Lviv Polytechnic National University. He is currently a PhD student with the Telecommunications Department, Lviv Polytechnic National University, Lviv, Ukraine. His research interests include optical transport networks, software-defined networks, Internet of Things, Big Data and artificial intelligence.

Stepan Dumych received his BS and MS in radio physics from Ivan Franko National University of Lviv, Ukraine, in 2004 and 2006, respectively. He is currently an Associate Professor with the Telecommunications Department, Lviv Polytechnic National University, Lviv, Ukraine. His research interests include Internet of Things, network func- tions virtualization, blockchain and artificial intelligence.

Bohdan Shubyn received the B.A. degree in telecommunications in 2018 and the M.S. degree in information communication networks in 2019, all from the Lviv Polytechnic National University. He is currently a PhD student with the Telecommunications Department, Lviv Polytechnic National University, Lviv, Ukraine. His research interests include $5 \mathrm{G}$ mobile networks, software-defined networks, blockchain and artificial intelligence.

Gabriel Bugár works as a Research Assistant with the Faculty of Electrical Engineering and Informatics, Technical University of Kosice, Slovakia. His research interests include cognitive networks, image processing, and e-learning.

Juraj Gazda is currently an Associate Professor with the Faculty of Electrical Engineering at the Technical University of Kosice, Slovakia. He was a Guest Researcher at Ramon Llull University, Barcelona, Spain, and worked under the supervision of Prof. Herman Rohling at the Technical University of Hamburg-Harburg, Germany. He was also involved in the development activities of Nokia Siemens Networks (NSN). In 2017 he was recognized as Best Young Scientist of the Technical University of Kosice. Currently, he serves as the editor of KSII Transactions on Internet and Information Systems and guest editor of Wireless Communications and Mobile Computing (Wiley). His research interests include spectrum pricing, techno-economic aspects of 5G networks, co-existence of heterogeneous networks, and application of machine learning principles in $5 \mathrm{G}$ networks. 